\title{
DOMAINS OF POSITIVITY
}

\author{
BY OSCAR S. ROTHAUS
}

Communicated by S. Bochner, February 10, 1958

A Domain of Positivity $D$ is an open convex cone associated with a nonsingular symmetric matrix $S$, called the characteristic, such that $x \in D$ if and only if $x^{\prime} S y>0$ for all $y \in \bar{D}$. As such they were introduced by Koecher (1) in generalization of the cone of positive definite matrices studied by Siegel. The automorphisms of $D$ are the nonsingular linear transformations mapping $D$ onto itself. The group of automorphisms $\{W\}$ admits an anti-automorphism: $W \rightarrow S^{-1} W^{\prime} S$, where $W^{\prime}$ means $W$ transposed. A norm $N(x)$ is a function positive and continuous for $x \in D$ and satisfying there $N(W x)=\|W\| N(x)$ for every automorphism $W$. A norm is given by:

$$
1 / N(x)=\int_{D} \exp \left(-x^{\prime} S t\right) d t
$$

and a group invariant positive definite metric form is given by:

$$
g_{i j}=-\frac{\partial^{2} \log N(x)}{\partial x_{i} \partial x_{j}} .
$$

The Domain is called homogeneous if the automorphisms are transitive. In this case the Domain has an involution given by:

$$
x \rightarrow x^{*}=S^{-1} \operatorname{grad} \log N(x) .
$$

For homogeneous domains it is easy to show that $N^{2}(x)$ is always a rational function. If the characteristic is positive definite much more is true. In the first instance, the fixed points of the anti-automorphism of the group of automorphisms already act transitively on the domain $D$. It follows that the norm satisfies the important equality:

$$
N\left(x^{*}+y^{*}\right) \cdot N(x) \cdot N(y)=N(x+y) .
$$

Moreover, for every point $x$ in $D$ there is an involution of the Domain keeping $x$ fixed. Hence $D$ is a symmetric (Cartan) space, and it is possible to make a detailed study of the Lie group of automorphisms. The following facts emerge:

(a) $N^{2}(x)$ is a polynomial,

(b) The geodesic connecting any two points (given by Cartan's construction of geodesics in a symmetric space) is unique, 
(c) The Domain has everywhere zero or negative curvature.

(d) The involution on $D$ extends to an analytic involution of the whole of the tube with $D$ as base onto itself.

On the basis of (a) it follows from a result of Bochner (2) that the so-called "Gamma-Factor" of the Domain is indeed a product of Gamma functions. From (d) together with the equality described above satisfied by the norm, it follows from another result of Bochner (3) that there exist unitary transformations of $L_{2}(D)$ relative to the volume element $N^{s}(x)$ for suitable values of $s$, which generalize the Hankel Transform.

In quite another direction is the result, true for any homogeneous domain that, except for a multiplicative constant, $1 / N^{2}(z+\bar{w})$ is the Bergmann reproducing kernel for analytic $L_{2}$ in the tube with $D$ as base. This result shows quite clearly that the involution on $D$ cannot extend to a holomorphic mapping of the tube with $D$ as base unless the norm satisfies the equality stated earlier.

We understand Koecher has proved some of the same results, though they are not yet published at this writing.

\section{REFERENCES}

1. Max Koecher, Positivitatsbereiche im $R^{n}$, Amer. J. Math. vol. 79, no. 3, July, 1957.

2. S. Bochner, Gamma factors in functional equations, Proc. Nat. Acad. Sci. vol. 42, no. 2, February, 1956.

3. - Bessel functions and modular relations of higher type and hyperbolic differential equations, Communications du Seminaire Mathematique de l'Universite de Lund, tome supplementaire, 1952, dedie a Marcel Riesz.

Princeton University 